\title{
A SIGNAL REGULARITY-BASED AUTOMATED SEIZURE PREDICTION ALGORITHM USING LONG-TERM SCALP EEG RECORDINGS ${ }^{1}$
}

Keywords: epileptic seizure, seizure warning, scalp electroencephalogram, brain dynamics.

\section{INTRODUCTION}

An epileptic seizure is a transient occurrence of signs and/or symptoms due to abnormal excessive and synchronous neuronal activity in the brain [1]. The worldwide prevalence of epilepsy ranges from $0.4 \%$ to $1 \%$ [2]. Some epileptic patients experience a prodrome or an aura [3], which can serve as a warning before frank signs of seizure onset. Rare patients learn to abort a seizure without external intervention [4]. However, most patients cannot predict or arrest their seizures. In industrialized countries, where antiepileptic drugs and seizure control devices are readily available, about $70 \%$ of epilepsy patients are able to gain satisfactory control of their seizures [5]. For patients whose seizures do not respond to antiepileptic medications, less than $50 \%$ are candidates for epilepsy surgery [6]. Therefore, approximately $15-20 \%$ of epilepsy patients have no choice but to live their lives with unforeseen and uncontrolled seizure attacks, which cause considerable stress for these patients and their care-givers and limit the range of daily activities available due to safety concerns. These lifestyle limitations decrease quality of life and may contribute to the increased prevalence of depression in patients with uncontrolled seizures [7]. If a device could be developed that could warn an epilepsy patient of an impending seizure, it could lessen the psychological stress of epilepsy and improve patient safety.

Results from several studies based on the analysis of intracranial EEG [8] and fMRI [9] data suggest the existence of a preictal transition between an interictal and ictal state. However, detecting a preictal transition using EEG signals from scalp electrodes may be more difficult due to the attenuating, spatial distortion, and filtering effects of the skull and soft tissues. Nevertheless, if a seizure warning system can be developed for scalp EEG, it could have a wide range of clinical diagnostic and therapeutic applications due to its portability, relatively low cost, and safety.

Scalp EEG has been used to investigate normal and pathological brain function for over 80 years, when Hans Berger published his work and coined the term "Elektenkephalogram" in 1929 [10]. Early attempts to predict seizures from EEG signals began in the 1970s and flourished during the 1990s [11]. Because of the non-linear nature of neuronal function and the paroxysmal character of a seizure, non-linear signal processing methods were popularly applied to EEG for predicting seizures [12-16]. By applying a non-linear similarity index, Le Van Quyen, Martinerie and colleagues studied preictal EEG dynamics in 26 scalp EEG recordings obtained from 23 patients with temporal lobe epilepsy. In five patients with simultaneously scalp and intracranial recordings, changes of similarity index values was observed

\footnotetext{
${ }^{1}$ This work was supported by the grants 5R01NS050582 (JCS) and 1R43NS064647 (DSS) from NIH-NINDS.

(C) Jui-Hong Chien, Deng-Shan Shiau, J.J. Halford, K.M. Kelly, R.T. Kern, M.C.K. Yang, Jicong Zhang, J.Ch. Sackellares, P.M. Pardalos, 2011
} 
during preictal period in both types of EEG recordings, and 25 out of the 26 EEG segments showed changes prior to the occurrence seizures (mean 7 minutes). Although this study did not evaluate the specificity of the similarity index change in long-term EEG recordings (contained only 50 minutes before seizure onsets), it rendered an encouraging result using only scalp EEG to achieve seizure prediction [17], and as is known, various prediction models were widely used in univariate and bivariate analysis [41]. Another study by Hively and Protopopescu used $L_{1}$-distance and $\chi^{2}$ statistic to estimate the dissimilarity in density functions between the base-windows and the test-windows in 20 scalp EEG recordings [18]. The result showed pre-seizure changes in all datasets with the forewarning times ranged from 10 to 13660 seconds. However, this study only examined one selected channel in each data set, and similar to the Le Van Quen study [17], specificity of the method was not reported. Two follow-up studies by the same group, one used all available recording channels and the other used a fixed channel, showed similar results $[19,20]$. While the results seemed promising, no validation study has been reported for this method. In the study reported by Corsini, Shoker and coworkers, 20 sets of simultaneously scalp and intracranial EEG recordings were analyzed using blind source separation and short-term Lyapunov exponent (STLmax) [12, 21]. They observed changes of STLmax values before seizure onsets and that the scalp EEG may give better predictive power over intracranial EEG when the intracranial electrodes did not record the electrical activity in the epileptic focus. However, the practicality of this method on long-term scalp EEG recordings may be limited due to the lack of an automatic procedure to select the most relevant source component. Schad et al. investigated seizure detection and prediction in 423 hours of long-term simultaneously scalp and intracranial EEG recordings from six epileptic patients [22]. The method used techniques based on simulated leaky integrate-and-fire neurons. The study reported that 59\% (50\%) of the 22 seizures were predicted using scalp (invasive) EEGs given a maximum number of 0.15 false predictions per hour. In the study by Bruzzo et al., a small sample of scalp EEG recordings (115 hours from 3 epileptic patients) was analyzed using permutation entropy (PE) [23]. By examining the area under the receiver operating characteristic (ROC) curve, they reported that the decrease of PE values was correlated with the occurrence of seizures. However, the authors also concluded that the dependency of PE changes on the vigilance state may restrict its possible application for seizure prediction. More recently, Zandi, Dumont and colleagues reported a prediction method based on the positive zero-crossing interval series [24]. The method was applied on a 21.5 hour scalp EEG dataset recorded from 4 patients with temporal lobe epilepsy. They reported a training result of $87.5 \%$ sensitivity (16 seizures) with a false prediction rate of 0.28 per hour, and the average prediction time was approximately 25 min. James and Gupta analyzed long-term continuous scalp EEG recordings from nine patients (5 in training set and the other 4 in test dataset) [25]. The data were processed by a sequence of techniques consisting of independent component analysis, phase locking value, neuroscale, and Gaussian mixture model. The prediction performance of this method achieved a sensitivity of $65-100 \%$ and specificity of $65-80 \%$ as the prediction horizon ranged from $35-65$ minutes in the test dataset.

One of the common features used in many of the seizure warning algorithms is the change of synchronization of EEG signals recorded from different electrode sites. The concept of "synchronization" can be quantified in several different ways such as coherence [26], phase synchronization [27], or entrainment of dynamic features (denoted as "dynamic entrainment" hereafter) [28]. In this study, we have attempted to identify preictal transitions by detecting dynamic entrainment based on the 
convergence of PMRS among multiple electrode sites. PMRS is a probabilistic statistic that quantifies the regularity of a time series. It is especially useful when the moment statistics (e.g., mean, variance, etc.) or frequency cannot detect changes in a signal. By further applying paired $t$-statistic (denoted as "T-index" hereafter) that quantifies the convergence of two PMRS time series over time, we constructed an automated seizure prediction algorithm that monitors the change of T-index and issues a warning of an impending when the T-index curve exhibits the pattern defined by the algorithm. The general hypothesis is that seizures are preceded by PMRS entrainment; this hypothesis was based upon findings reported previously using a different measure of signal order, STLmax $[12,29-31,40]$. The prediction parameters and the specific EEG channels to be monitored were determined by the use of a training dataset. Algorithm performance was then assessed using an independent test dataset. The performance was further validated by comparison with that from a random warning scheme that did not use any information from the EEG signals.

\section{METHODS}

\subsection{Data Characteristics}

2.1.1. Subjects and EEG recording specifications. All subjects were 18 years of age or older admitted to either Allegheny General Hospital (AGH, Pittsburgh, PA) or the Medical University of South Carolina (MUSC, Charleston, SC) for inpatient seizure monitoring for diagnostic purposes or presurgical evaluation. Data collection procedure was approved by the Investigational Review Boards of AGH and MUSC, and the Western Investigational Review Board (WIRB). The EEG recordings at MUSC were obtained using XLTEK monitoring systems (Oakville, Ontario, Canada) with a sampling rate of $256 \mathrm{~Hz}$ and the EEG recordings at AGH used 128-channel Nicolet BMSI-6000 systems (Viasys, Madison, WI, USA) with a $400 \mathrm{~Hz}$ sampling rate. The EEGs recorded at both institutions used a referential montage and the 19-electrode international 10-20 system of electrode placement. The exact locations of referential electrodes placed in our dataset were decided on-site and usually followed the recommended location of the $\mathrm{Cz}$ and $\mathrm{Pz}$ electrodes as suggested by the American Clinical Neurophysiology Society [32]. All segments were reviewed by the collaborative clinical sites (AGH and MUSC) and all seizure events were verified by $\mathrm{KK}$ and $\mathrm{JH}$, respectively.

2.1.2. Data selection. Because a scalp EEG might be severely contaminated with artifact, typical muscle contraction and movement artifacts include blinking, chewing, and talking, only EEG segments with a tolerable level of artifact, i.e., not present for more than $50 \%$ of the recording and not involving more than $50 \%$ of the recording channels, were included in this study. All EEG segments were long-term recordings (mean $=24.4$ hours) containing at least one seizure. EEG segments containing more than one seizure within any two-hour interval were excluded to avoid potential overlap between preictal and postictal periods of consecutive seizures. Thus, the warning algorithm had a sufficiently long period of observation to detect the transition from interictal to ictal states. The resultant dataset of EEG recordings included in this study was collected from 52 patients. The dataset was randomly divided into training $(n=21)$ and test $(n=31)$ sets. Both training and test sets had more than 40 seizures (43 and 60, respectively). Subjects from each clinical site had a similar proportion of seizures in the two datasets. The individual recording durations of each subject in the training dataset and test dataset are shown in Fig. 1 and Fig. 2, respectively. The mean recording duration for each subject in the training dataset was 39.78 hours and that in the test dataset was 33.54 hours. The standard deviation of recording duration was 22.96 hours in the training dataset and 15.99 hours in the test dataset. The shortest recording duration was 6.18 hours and the longest duration was 92.41 hours. 


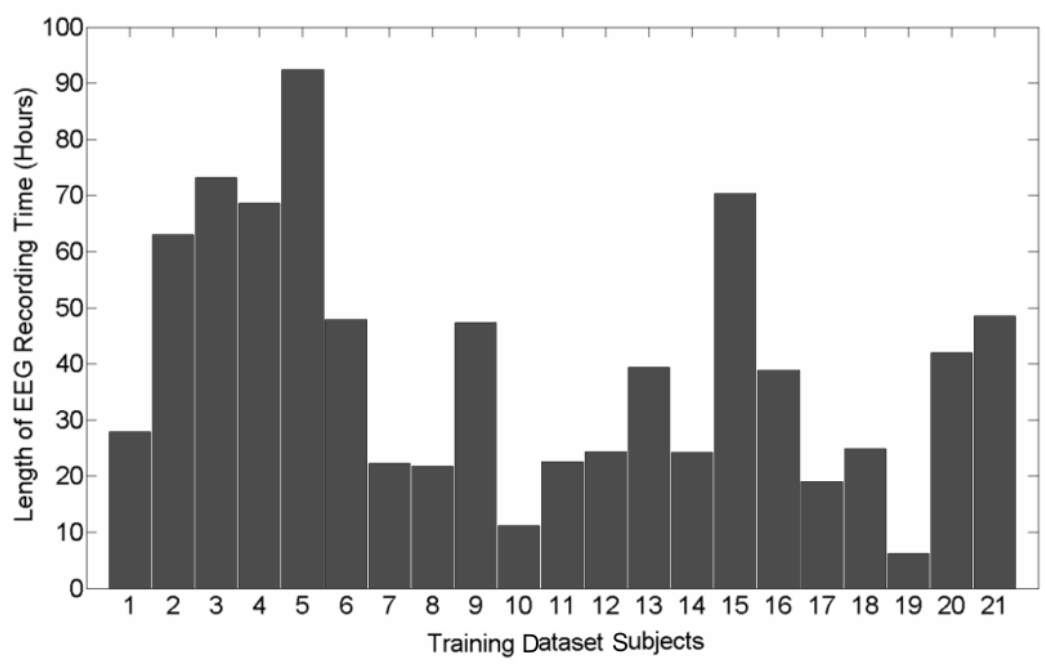

Fig. 1. Recording duration of all 21 subjects in the training dataset. The range of recording duration was between 6.18 to 92.41 hours. The mean was 39.78 hours and the standard deviation was 22.96 hours

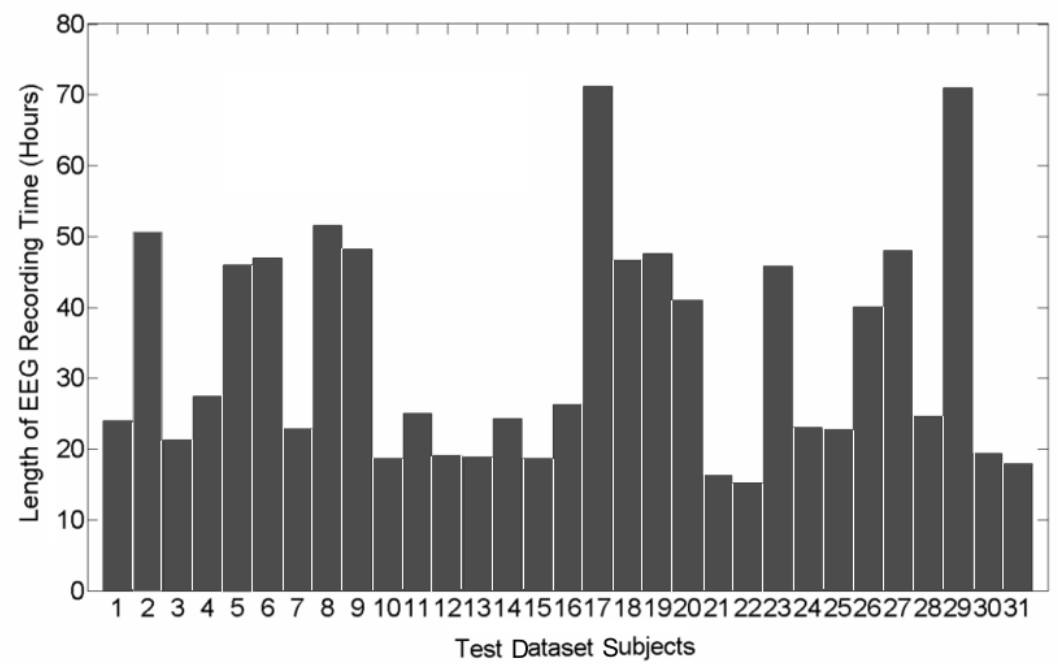

Fig. 2. Recording duration of each subject in the test dataset. The range of recording duration was between 15.16 to 71.21 hours. The mean was 33.54 hours and the standard deviation was 15.99 hours

\subsection{Pattern-Match Regularity Statistic (PMRS)}

PMRS is a probabilistic statistic quantifying signal regularity [33, 34]. One of the characteristic features of EEG signals during a seizure is the rhythmic and regular discharges over a wide range of the brain. Therefore, the first step of the warning algorithm presented in this study calculated PMRS sequentially for each EEG signal analyzed. The rationale of applying this pattern match method (instead of value match) is due to its robustness over scalp signal values, which are usually more unstable than their up-and-down trends. The procedure for calculating PMRS is described below.

Given a time series $U=\left\{u_{1}, u_{2}, \ldots, u_{n}\right\}$ with standard deviation $\hat{\sigma}_{n}$, a tolerance coefficient $e$, and a fixed integer $m$, the two segments in $U\left(x_{i}=\left\{u_{i}, u_{i+1}, \ldots, u_{i+m-1}\right\}\right.$, $\left.x_{j}=\left\{u_{j}, u_{j+1}, \ldots, u_{j+m-1}\right\}\right)$ are considered pattern-matched to each other when: 


$$
\begin{gathered}
\left\{\left|u_{i}-u_{j}\right| \leq e \hat{\sigma}_{n}\right\} \wedge\left\{\left|u_{i+m-1}-u_{j+m-1}\right| \leq e \hat{\sigma}_{n}\right\} \wedge \\
\wedge\left\{\operatorname{sign}\left(u_{i+k}-u_{i+k-1}\right)=\operatorname{sign}\left(u_{j+k}-u_{j+k-1}\right)\right\}, \quad k=1,2, \ldots, m-1 .
\end{gathered}
$$

The first two criteria require value match to some extent at both the beginning and ending points of two segments, where $e$ was set to be 0.2 empirically. The third criterion requires pattern match between $x_{i}$ and $x_{j}$ within a range of $m$ (set as 3 in this study). To calculate PMRS, we first define a conditional probability:

$$
\begin{gathered}
p_{i}=\operatorname{Pr}\left\{\operatorname{sign}\left(u_{i+m}-u_{i+m-1}\right)=\right. \\
\left.=\operatorname{sign}\left(u_{j+m}-u_{j+m-1}\right) \mid x_{i} \text { and } x_{j} \text { are pattern match }\right\} .
\end{gathered}
$$

Given $m, p_{i}$ can be estimated as: for $1 \leq j \leq n-m$,

$\hat{p}_{i}=\frac{\left.\# \text { of }\left\{x_{j}^{\prime} \text { s pattern match with } x_{i}\right] \wedge\left[\operatorname{sign}\left(u_{i+m}-u_{i+m-1}\right)=\operatorname{sign}\left(u_{j+m}-u_{j+m-1}\right)\right]\right\}}{\# \text { of }\left\{x_{j}^{\prime} \operatorname{s~pattern~match~with~} x_{i}\right\}}$,

where $1 \leq i \leq n-m$, and finally,

$$
\mathrm{PMRS}=-\frac{1}{n-m} \sum_{i=1}^{n-m} \ln \left(\hat{p}_{i}\right)
$$

As the time series $U$ develops into a more regular state, $\hat{p}_{i} \mathrm{~s}$ become larger and PMRS decreases as a result.

\subsection{PMRS Entrainment (T-index)}

T-index is basically the paired $t$-statistic function used to quantify the degree of entrainment between two PMRS time series. The specific calculation is as follows.

For two time series $X_{i}$ and $X_{j}$ (the PMRS value time series), if their values in a calculation window $W^{t}$ with a size of $n$ data points are presented as:

$$
\begin{aligned}
& L_{i}^{t}=\left\{X_{i}^{t}, X_{i}^{t+1}, \ldots, X_{i}^{t+n-1}\right\}, \\
& L_{j}^{t}=\left\{X_{j}^{t}, X_{j}^{t+1}, \ldots, X_{j}^{t+n-1}\right\}
\end{aligned}
$$

then the pair-wise differences between $L_{i}^{t}$ and $L_{j}^{t}$ can be written as:

$$
\begin{gathered}
D_{i j}^{t}=L_{i}^{t}-L_{j}^{t}=\left\{X_{i}^{t}-X_{j}^{t}, X_{i}^{t+1}-X_{j}^{t+1}, \ldots, X_{i}^{t+n-1}-X_{j}^{t+n-1}\right\}= \\
=\left\{d_{i j}^{t}, d_{i j}^{t+1}, \ldots, d_{i j}^{t+n-1}\right\} .
\end{gathered}
$$

The T-index over the calculation window $W^{t}$ between the two time series is calculated by:

$$
\operatorname{Tind}_{i j}^{t}=\left|\bar{D}_{i j}^{t}\right| / \frac{\hat{\sigma}_{D_{i j}^{t}}}{\sqrt{n}},
$$

where $\bar{D}_{i j}^{t}$ and $\hat{\sigma}_{D_{i j}^{t}}$ are the sample mean and the sample standard deviation of $D_{i j}^{t}$, respectively. PMRS entrainment is a process during which one EEG signal is influenced by or coupled with another with respect to the signal regularity. This phenomenon was used as a dynamical pattern for warning of an impending seizure. Fig. 3 shows the PMRS traces derived from three EEG channels (F8, T4, and T6) (upper panel) and their average T-index values (bottom panel) over a 350-minute interval containing a seizure. As shown in the PMRS plot, all PMRS values of the 
three channels dropped seconds after the seizure onset (indicated by a black vertical line), which was due to the extreme signal singularity during the ictal period. More importantly, the PMRS values became convergent about $60 \mathrm{~min}$ before the seizure onset, which caused the decrease of T-index values (shown in T-index plot).

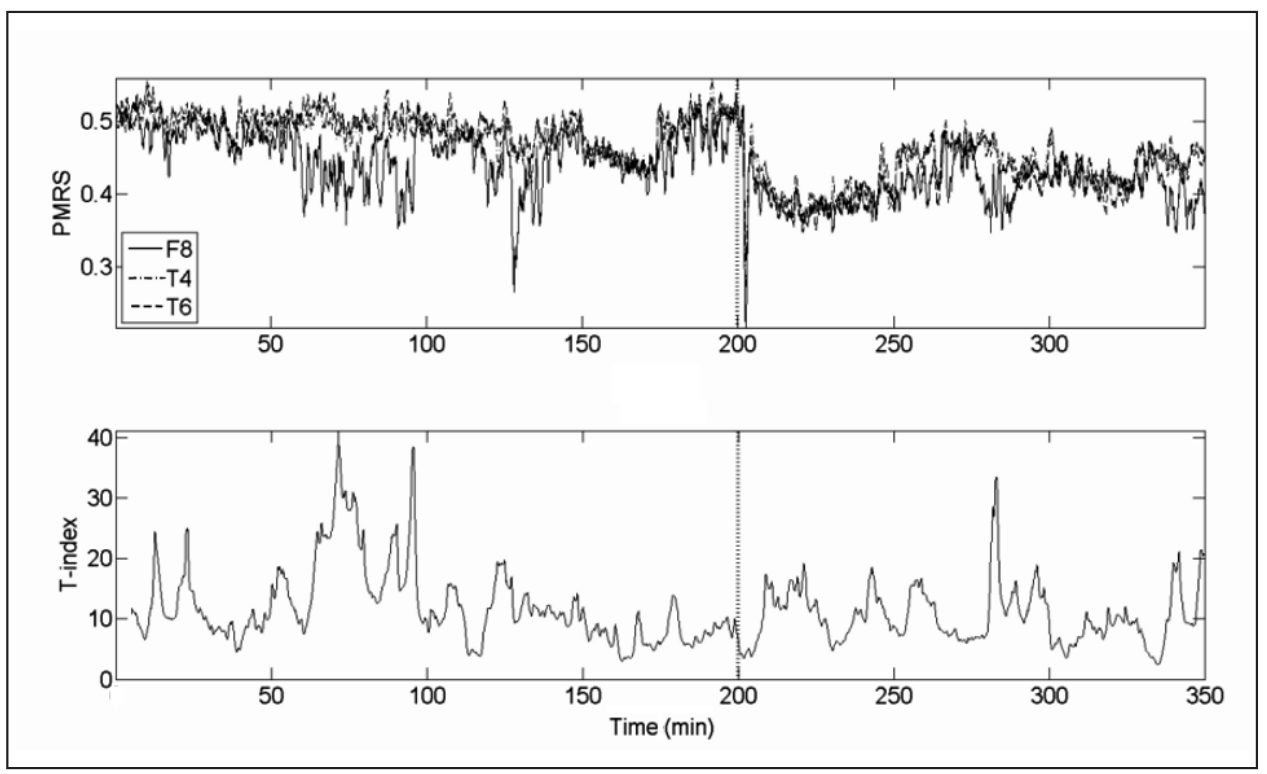

Fig. 3. Dynamic features of three EEG electrode signals. Top: PMRS traces of F8, T4, and T6 electrodes. There is a sudden drop of PMRS values in all three channels right after the seizure onset (denoted by vertical dashed black lines in both panels at the 200-min time point). Bottom: averaged T-index among the three channels. Approaching the time of seizure occurrence, a gradual decrease of the T-index (entrainment) from approximately the 120 -min point to the 160 -min point can be observed. The T-index values remain small before the seizure

\subsection{Seizure Warning Mechanism}

2.4.1. Overview. EEG signals were first filtered by a fifth-order Butterworth filter with a band-passing frequency between 1 to $20 \mathrm{~Hz}$ (the bandwidth within which most ictal epileptiform patterns occur) [35]. After the filtering process, for each channel, PMRS was calculated for each non-overlapping 5.12-second epoch. Based on the PMRS values, T-indices were then calculated for each of the selected channel groups (three channels each). To increase the sensitivity of seizure warning, the proposed algorithm independently monitored four T-index curves (i.e., from four channel groups). A warning was issued when any of the monitored T-index curves met entrainment criteria.

2.4.2. Selection of channel groups forseizure warning monitoring. Channel groups were selected such that they were bilaterally symmetric along the midsagittal line. In order to avoid frequent eye movement artifact, electrodes Fp1 and Fp2 were excluded. In addition, to avoid the regular alpha rhythm pattern that might give potential false positive warnings because of the nature of the regularity statistics used in our prediction algorithm, electrodes $\mathrm{O} 1$ and $\mathrm{O} 2$ were also excluded.

The four channel groups selected in this study resemble the well known and popular anterior-posterior bipolar ("double banana") montage. These four channel groups were: (F7, T3, T5), (F3, C3, P3), (F4, C4, P4), and (F8, T4, T6). The main rationale for this selection was that most seizures occurring in patients with temporal lobe epilepsy start from a channel group on one side (hemisphere) of the brain, and therefore, intuitively, these channels were more likely to be entrained with each other 
during the preictal transition period. By monitoring four channel groups that cover both hemispheres, the algorithm was enabled to detect a PMRS entrainment that preceded an impending seizure initiated from either hemisphere.

For each channel group $k, k=1,4$, the group T-index over the calculation window $W^{t}$ is denoted as:

$$
\text { GTind }_{k}^{t}=\frac{\left\{\sum_{i=1}^{2} \sum_{i^{\prime}=i+1}^{3} \text { Tind }_{i i^{\prime}}^{t}\right\}}{3} \text {. }
$$

The window size for calculating one group T-index is 60 PMRS data points, with 59 points overlapping from $t$ to $t+1$ (i.e., sliding window). The warning algorithm only monitored the four group T-index curves instead of individual pair-wise T-indices.

2.4.4. Detection of PMRS entrainment. The proposed prediction algorithm, instead of attempting to detect a signal threshold crossing, was set to detect a certain pattern of T-index dynamics that gradually descends from a baseline value. Therefore, the first step to detect such a pattern was to determine an upper threshold $U_{T}$ (i.e., baseline value, see an illustration in Fig. 4). A decrease of a group T-index from $U_{T}$ was considered as a necessary condition for a potential PMRS entrainment, and an entrainment was identified when the group T-index values fell below a lower threshold $L_{T}$. It was further defined that a period of entrainment should be maintained for at least several minutes. For each group T-index, its $U_{T}$ was set to be the asymptotic 95th percentile in the preceding $12 \mathrm{~min}$, as described in equation 10 . The duration of $12 \mathrm{~min}$ was decided by training across multiple patients in the training dataset:

$$
U_{T}=\operatorname{mean}\left(T_{12 \min }(t)\right)+2 \times \operatorname{std}\left(T_{12 \min }(t)\right) .
$$

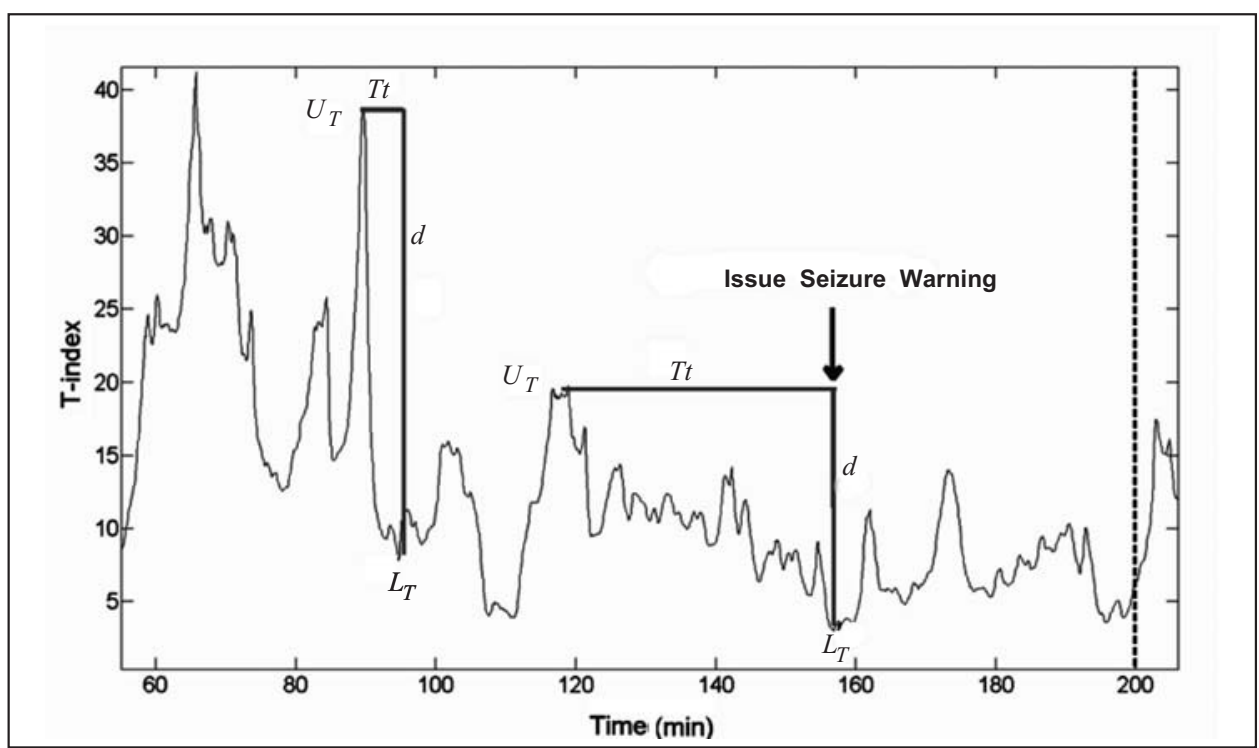

Fig. 4. Resized plot of Fig. 3 with warning algorithm parameter indications. The seizure warning sensitivity relates to parameters $t_{t}$ and $D$. As the algorithm finds both $L_{T}$ and $U_{T}$, the difference of time and the group T-index between them, as indicated by $T t$ and $d$ in the figure, are compared with $t_{t}$ and $D$, respectively. Only the decrease $d$ of a T-index curve was larger than $D$, and also $t t$ larger than $t_{t}$ would trigger a seizure warning. For example, with the proper setting of $t_{t}$ and $D$, the T-index drop at $100 \mathrm{~min}$ was not considered a warning event because a large $d$ occurred during a short Tt. However, the T-index drop from the 120 -min point to the 160 -min point shows a gradual and persistent decrease and is considered a proper warning event. The dashed black line denoted the seizure onset time point 
However, if half of the following 16 T-index values (representing 81.92 seconds of data) were greater than $U_{T}, U_{T}$ was updated as the median of these 16 values. This operation is described in equations 11 and 12 :

$$
\begin{gathered}
U_{T}=U_{T} \times(1-I(A))+\operatorname{median}(T) \times I(A), \\
A=\left\{\sum_{k=1}^{16} H\left[T(t+K)-U_{T}\right]>\frac{16}{2}\right\},
\end{gathered}
$$

where $I(\cdot)$ is an indicator function, $A$ is the criterion of updating $U_{T}$, and $T(t)$ is the group T-index value at time $t$. If $\mathrm{A}$ is false, then $U_{T}$ is kept as it is. $H(\cdot)$ denotes the Heaviside step function. $U_{T}$ is not updated with a maximum value because the existence of artifact in raw EEG recordings could affect the T-index causing an abrupt surge and therefore produce an abnormally high $U_{T}$. Once $U_{T}$ is determined, $L_{T}$ is equal to $U_{T}$ minus $D$, where $D$ is a parameter that would determine the sensitivity of the algorithm. In general, the bigger $D$ is, the less sensitive the algorithm will be, but the less susceptible the algorithm will be to false warnings.

In addition to the above detection rules, the period of a descending $\mathrm{T}$-index pattern from $U_{T}$ to $L_{T}$ must continue for more than $t_{t}$ minutes to be regarded as a PMRS entrainment. Therefore, the algorithm would issue a warning of an impending seizure only when any of the monitored T-index curves traveled from $U_{T}$ to $L_{T}$ with the traveling time longer than $t_{t}$ to ensure a gradual descendent pattern.

Once an entrainment was identified, other entrainments that followed (identified within the seizure warning horizon (SWH) by the warning algorithm using the same parameter settings) were silenced due to the possibility that an entrainment preceding an onset may be much longer than the $t_{t}$ value and cause several entrainment identifications.

The following flow chart gives an overview of our seizure warning algorithm.

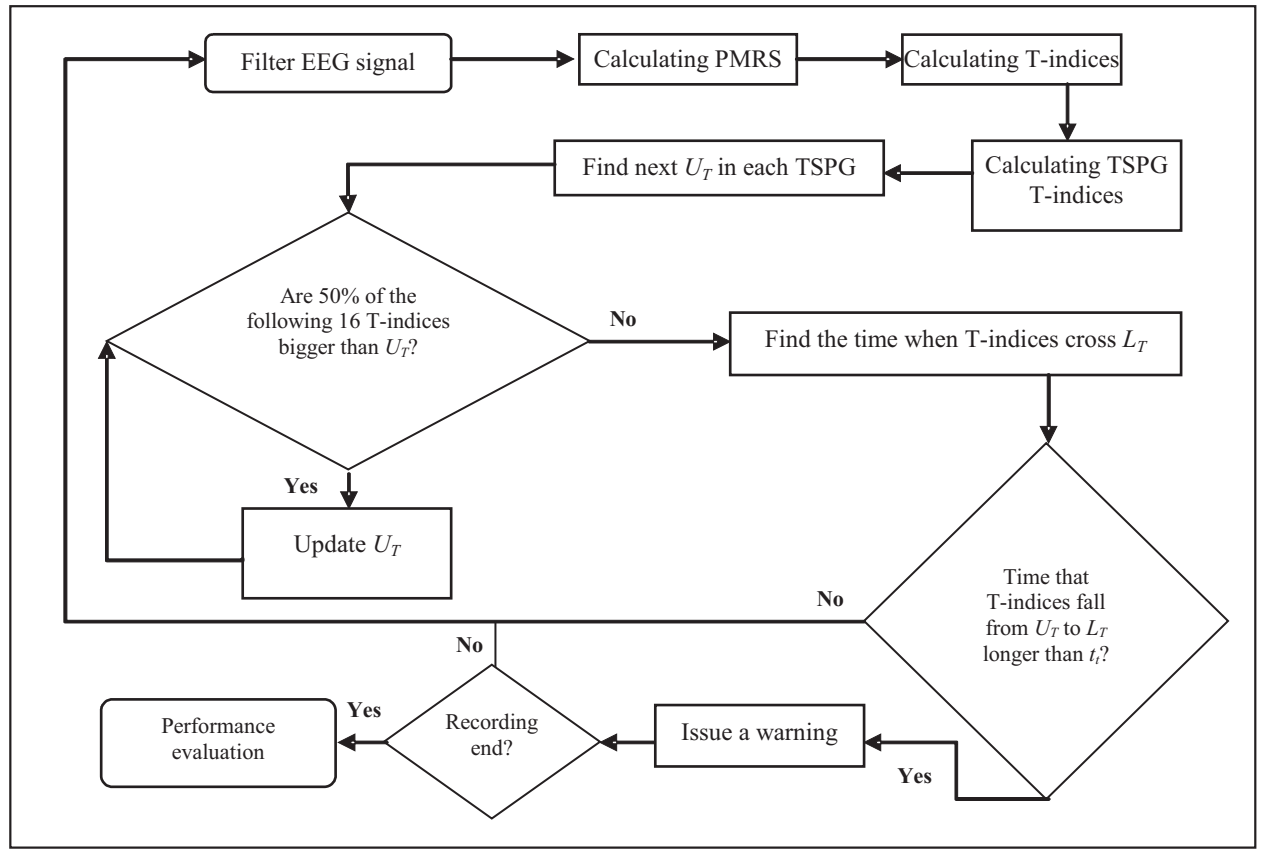

Fig. 5. Flow chart of the seizure warning mechanism 


\subsection{Statistical Evaluation}

2.5.1. Estimation of performance statistics. The performance of the seizure warning algorithm varies with the length of the seizure warning horizon $(\mathrm{SWH})$ - the longer the SWH, the lower the sensitivity and false positive rate (FPR). In this work, SWH was defined as the time window following a seizure warning during which the patient was likely to have a seizure [31]. It is worth noting that Winterhalder et al. [36] defined the SWH (same as seizure prediction horizon, SPH) differently as a short intervention preparation period. The definition of SWH here is closer to the "seizure occurrence period" defined by this group. A seizure warning was considered true only when at least one seizure onset occurred within the SWH following the warning. Otherwise, the warning was considered a false positive.

After determining the outcome (i.e., true or false) of each warning, sensitivity was estimated by dividing the number of correct warnings by the total number of seizures in the EEG segments, i.e. the proportion of seizures correctly predicted (within SWH). FPR (per hour) was calculated by dividing the total number of false positives by the total recording hours outside the SWH before each seizure. The SWH period before each seizure was excluded because it was impossible for a false warning to occur within the SHW before each seizure.

2.5.2. Statistical validation - comparison with a random seizure warning scheme. Several methods have been proposed for validating the performance of a seizure warning algorithm [37-39]. Each study tested a specific statistical hypothesis. In this study, we compared the prediction sensitivity estimates with a random prediction scheme that issued random seizure warnings (in time) during the recording. Under this random scheme, the interval between two consecutive warnings followed an exponential distribution, with a condition that the length of any interval could not be smaller than the SWH. This additional condition ensured that the two compared algorithms (test algorithm and prediction scheme) were consistent in terms of the management of nearby warnings (within the SWH). Although this condition diminished some randomness from the random prediction scheme, it was necessary in order to have a meaningful comparison of prediction performance between the two prediction methods.

In order to have an unbiased comparison for sensitivity, two parameters were controlled: 1) length of the SWH, and 2) total number of warnings. For each of the test EEG segments, the total number of warnings allowed by the random predictor was set to be the same as that issued by the test algorithm. For example, if the test algorithm issued two true positive and two false warnings in a specific segment, the random predictor would only randomly issue a total of four warnings. Under the same number of warnings allowed, the study compared overall prediction sensitivity across all test patients. A distribution of overall sensitivity by the random prediction scheme was generated from 1000 simulations, and was used to assess how significant the prediction performance by the test algorithm was over the random scheme.

It is worth mentioning that it was necessary to impose the above conditions in the random warning algorithm compared in this study to make it comparable with the proposed algorithm. Since this was a conditioned random process, the specific null hypothesis tested in this study was: "The overall sensitivity (or FPR) of the test algorithm is the same as that of a random prediction scheme with the same number of warnings issued by the test algorithm."

\section{RESULTS}

\subsection{Training Results}

The proposed algorithm was applied on the training dataset $(n=21)$ to optimize settings for the parameters $D$ and $t_{t}$. Parameter $D$ sets the minimal requirement that a group T-index value needs to decrease from $U_{T}$ to be considered as a PMRS entrainment, and $t_{t}$ gives the constraint on the minimal time length for a T-index curve traveling from $U_{T}$ to $L_{T}$. The training results are presented in Fig. 6, which shows the receiver operating characteristic (ROC) curves (sensitivity vs. false 
positive rate) under different $t_{t}$, ranging from 10 to 60 minutes with 10 -minute increments. Each ROC curve contains eight settings of parameter $D$, each representing the performance under a certain $D$ value, ranging from 1 to 8 (in T-index units) with increments of 1 . As $D$ increased, each ROC curve first reached its peak sensitivity and then declined as $D$ kept increasing. This was due to the constraint of $t_{t}$ : when $D$ was set to a small value, $T_{t}$, the time interval during which a T-index curve drops from $U_{T}$ to $L_{T}$, was likely to be shorter than the minimally required $t_{t}$, and therefore the drop of the T-index curve could not be considered as a PMRS entrainment. As $D$ became bigger, the sensitivity started increasing until the $D$ value became too large to start affecting the number of T-index drops (i.e., sensitivity started to reduce).

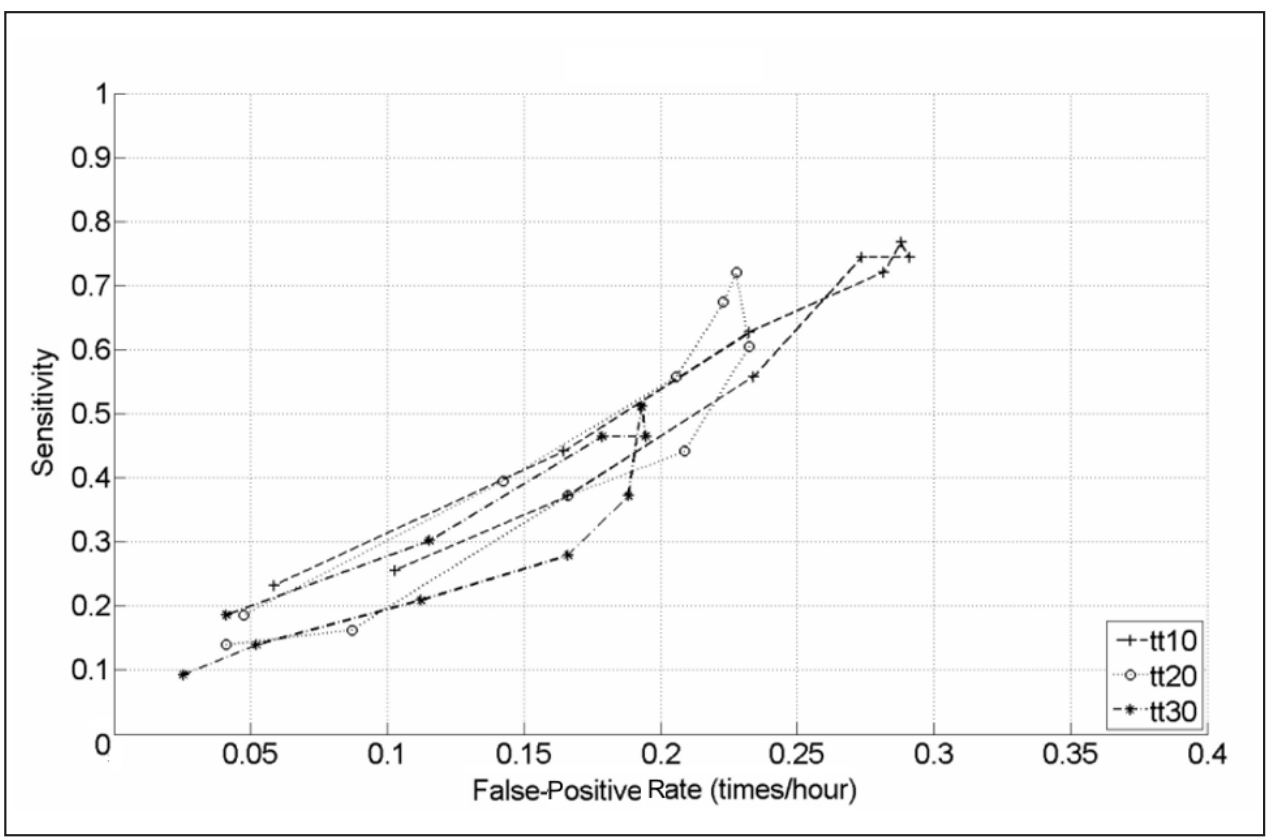

Fig. 6. Training results. ROC curves are shown under different $t_{t}$ values $\left(t_{t}=40,50,60\right.$ results are not shown in this figure). Each point in the ROC curve corresponds to an overall result over the entire training dataset with a specific $D$ value. The inset indicates the parameter $t_{t}$ used in each ROC curve. For example, tt10 denotes the ROC curve using parameter $t_{t}=10 \mathrm{~min}$. The best parameter configuration was observed at the $t_{t}$ value equal to $20 \mathrm{~min}$, which achieved a sensitivity above 0.7 with a false positive rate of 0.225 per hour

In this training study, parameters were optimized such that the test algorithm achieved sensitivity of at least $70 \%$ with the lowest possible false positive rate. As a result, the best prediction performance was obtained when tt was set to 20 minutes and $D$ was equal to $6 \mathrm{~T}$-index units. These parameters were fixed for the performance evaluation in the test dataset.

\subsection{Test Results}

The test dataset consisted of long-term EEG segments from 31 patients with a total of 60 seizures. With the parameter settings determined in the training dataset, the test algorithm gave 41 correct warnings among these segments. Using the prediction parameters obtained in the training study, the overall sensitivity was $68.3 \%$ with an overall false positive rate of 0.235 per hour.

The random warning scheme was designed to issued random warnings with the same number as that issued by the test algorithm for each segment in the test dataset. Each random trial ran through all of the patients in the test dataset, and an overall sensitivity was calculated. The random scheme repeated 1000 times on the test dataset and thus generated 1000 overall sensitivity estimates. Its (empirical) distribution is shown in Fig. 7. 


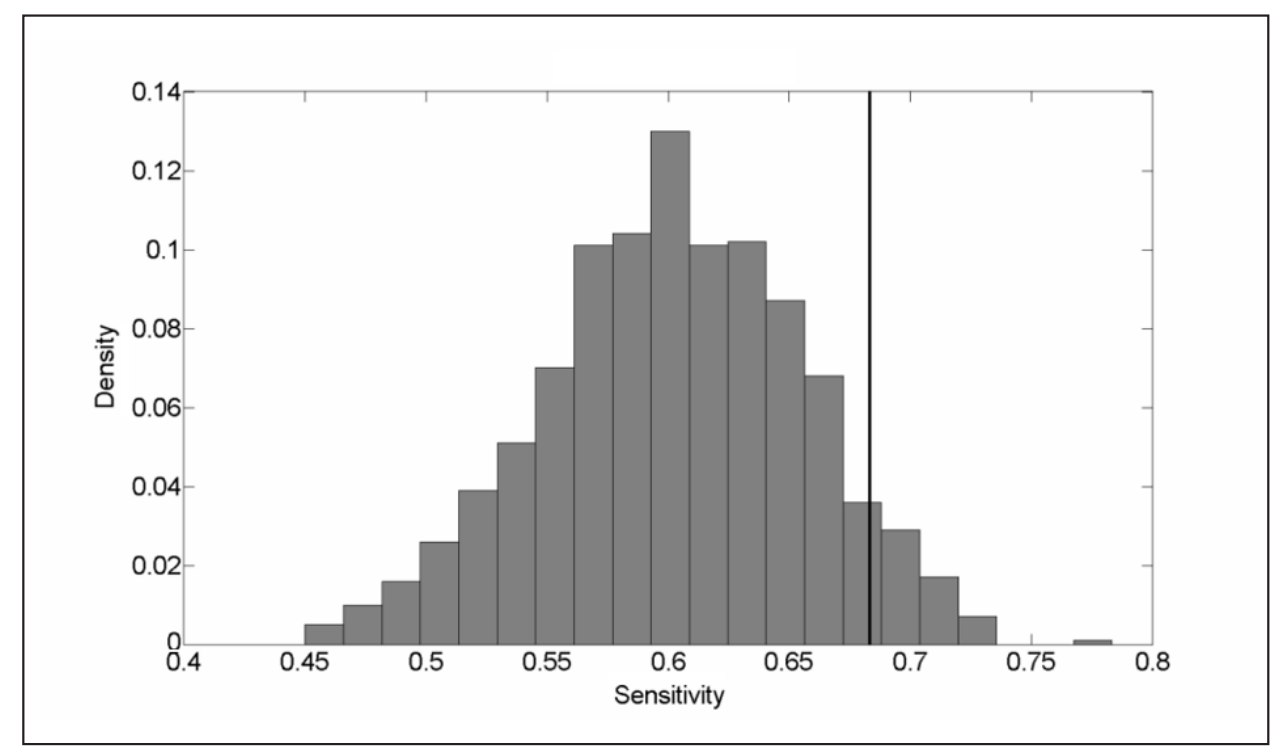

Fig. 7. The distribution of the overall sensitivities achieved by the random warning scheme (generated from 1000 trials). The overall sensitivity of the test algorithm is denoted by the dashed black vertical line, which was better than $91 \%$ of the trials by the random scheme

The overall sensitivity achieved by the test algorithm was then compared with the sensitivity distribution of the random warning scheme. The sensitivity of the test algorithm exceeded the sensitivity of $91 \%$ of the random warning trials $(p$-value $=0.09)$. Fig. 7 also presents this comparison.

\section{DISCUSSION}

This paper presents an automated algorithm that issues seizure warnings by monitoring the convergence of signal regularity among EEG channels of continuous long-term scalp EEG recordings. The algorithm was developed with a training dataset and its performance was evaluated using a separate test dataset. In the test dataset, the algorithm achieved a sensitivity of $68 \%$ with a false positive rate of 0.235 per hour. The algorithm performance in the test dataset was nearly the same as that in the training dataset. This implies that the algorithm generated stable performance on epileptic patients. The overall sensitivity achieved by the test algorithm was better than a random warnings process with a $91 \%$ confidence level $(p$-value $=0.09)$. While these results are encouraging, the performance of the algorithm may not be sufficient for some clinical applications for which greater sensitivity or lower false positive rates are required. In our study, we use a single parameter setting for all patients. In a real world application, however, such as control devices, it may be possible to tune the algorithm, by adjusting specific parameters to achieve better results in individual patients. For example, the vagus nerve stimulator (VNS) stimulation parameters are adjusted on a patient-by-patient basis, as is the deep brain stimulator (Medtronic) for Parkinson's disease. Similarly, the Neuropace seizure control device is adjusted with respect to epileptiform discharge detections on a patient-by-patient basis, to achieve the best results possible for each patient. In that sense, the study in this paper is proof of concept, demonstrating that prediction from scalp EEG is possible.

The result of this study should not be interpreted to implythat the performance of the proposed algorithm outperformed any random prediction scheme. As mentioned in section 2.5.2, the study tested a specific null hypothesis based on the random prediction scheme designed. In fact, a comparison of performance with a completely random prediction scheme may have very little significance, both scientifically and practically. Certain conditions should be imposed on the random scheme to prevent potential bias in the comparison, especially for the assessment of a sophisticated 
EEG-based prediction algorithm. Nevertheless, there are several studies that have applied different random warning schemes or surrogate data to test different hypotheses [22, 37-39]. The comparison of the proposed algorithm with other random warning schemes should be followed. Furthermore, studies on a larger sample size and the assessment of the proposed algorithm with different SWHs should be completed in the future.

\section{REFERENCES}

1. Epileptic seizures and epilepsy: definitions proposed by the International League Against Epilepsy (ILAE) and the International Bureau for Epilepsy (IBE) / R.S. Fisher, W.E. Boas, W. Blume, C. Elger, P. Genton, P. Lee and J. Engel // Epilepsia. - 2005. - 46. - P. 470-472.

2. S a nder J.W. The epidemiology of epilepsy revisited // Curr. Opin. Neurol. — 2003. — 16. P. $165-170$.

3. Gupta A.K., Jeavons P.M., Hughes R.C., Covanis A. Aura in temporal lobe epilepsy: clinical and electroencephalographic correlation // Br. Med. J. - 1983. - 46. P. 1079-1083.

4. Lee S.A., No Y.J. Perceived self-control of seizures in patients with uncontrolled partial epilepsy // Seizure: European J. of Epilepsy. — 2005. - 14. — P. 100-105.

5. Kwan P., Brodie M. J. Early identification of refractory epilepsy // N. Engl. J. Med. 2000. - 342. - P. 314-319.

6. Engel J., Shew mon D. A. Overview: who should be considered a surgical candidate // In Surgical Treatment of the Epilepsies / Ed. by J. Engel. — New York: Raven Press, 1993. — P. 23-34.

7. Kanner A.M. Depression in epilepsy: prevalence, clinical semiology, pathogenic mechanisms, and treatment // Biol. Psychiatry. - 2003. - 54. - P. 388-398.

8. Preictal state identification by synchronization changes in long-term intracranial EEG recordings / M. Le Van Quyen, J. Soss, V. Navarro, R. Robertson, M. Chavez, M. Baulac and J. Martinerie // Clinical Neurophysiology. - 2005. - 116. - P. 559-568.

9. Function al MRI of the pre-ictal state / P. Federico, D.F. Abbott, R.S. Briellmann, A.S. Harvey and G.D. Jackson // Brain. - 2005. - 128. - P. 1811-1817.

10. Swartz B.E., Goldensohn E.S. Timeline of the history of EEG and associated fields // Electroencephalogr. Clin. Neurophysiol. - 1998. - 106. - P. 173-176.

11. Lehnertz K., Litt B. The first international collaborative workshop on seizure prediction: summary and data description // Clinical Neurophysiology. - 2005. - 116. - P. 493-505.

12. Iasemidis L.D., Sackellares J.C., Zaveri H.P., Williams W.J. Phase space topography and the Lyapunov exponent of electrocorticograms in partial seizures // Brain Topogr. 1990. - 2. - P. 187-201.

13. Hughes J.R. Progress in predicting seizure episodes with nonlinear methods // Epilepsy and Behavior. - 2008. - 12. - P. 128-135.

14. Osterhage H., Lehnertz K. Nonlinear time series analysis in epilepsy // International J. of Bifurcation and Chaos. - 2007. - 17. - P. 3305-3323.

15. Osterhage H., Mormann F., Staniek M.A.U., Lehnertz K. Measuring synchronization in the epileptic brain: a comparison of different approaches // International J. of Bifurcation and Chaos. - 2007. - 17. - P. 3539-3544.

16. L e hnertz K. Epilepsy and nonlinear dynamics // J. Biol. Phys. — 2008. — 34. — P. 253-266.

17. Anticipation of epileptic seizures from standard EEG recordings / M. Le Van Quyen, J. Martinerie, V. Navarro, P. Boon, M. D'Havé, C. Adam, B. Renault, F. Varela and M. Baulac // The Lancet. - 2001. - 357. - P. 183-188.

18. Hively L.M., Protopopescu V.A., Gailey P.C. Timely detection of dynamical change in scalp EEG signals // Chaos: An Interdisciplinary J. of Nonlinear Science. — 2000. — 10. - P. 864-875.

19. Protopopescu V.A., Hively L.M., Gailey P.C. Epileptic event forewarning from scalp EEG // J. of Clinical Neurophysiology. - 2001. - 18. - P. 223-245.

20. Hively L.M., Protopopescu V.A. Channel-consistent forewarning of epileptic events from scalp EEG // IEEE Trans. on Biomed. Eng. — 2003. - 50. — P. 584-593.

21. Corsini J., Shoker L., Sanei S., Alarcon G. Epileptic seizure predictability from scalp EEG incorporating constrained blind source separation // IEEE Trans. on Biomed. Eng. 2006. - 53. - P. 790-799.

22. Application of a multivariate seizure detection and prediction method to non-invasive and intracranial long-term EEG recordings / A. Schad, K. Schindler, B. Schelter, T. Maiwald, A. Brandt, J. Timmer and A. Schulze-Bonhage // Clin. Neurophysiol. - 2008. - 119. - P. 197-211. 
23. P e rmutation entropy to detect vigilance changes and preictal states from scalp EEG in epileptic patients. A preliminary study / A.A. Bruzzo, B. Gesierich, M. Santi, C.A. Tassinari, N. Birbaumer and G. Rubboli // Neurol. Sci. - 2008. - 29. - P. 3-9.

24. Zandi A.S., Dumont G.A., Javidan M., Tafreshi R. An entropy-based approach to predict seizures in temporal lobe epilepsy using scalp EEG // Conf. Proc. IEEE Eng. Med. Biol. Soc. - 2009. - P. 228-231.

25. James C.J., Gupta D. Seizure prediction for epilepsy using a multi-stage phase synchrony based system // Conf. Proc. IEEE Eng. Med. Biol. Soc. - 2009. - P. 25-28.

26. Measuring the coherence of intracranial electroencephalograms / H.P. Zaveri, W.J. Williams, J.C. Sackellares, A. Beydoun, R.B. Duckrow and S.S. Spencer // Clin. Neurophysiol. — 1999. — 110. - P. 1717-1725.

27. A u t o m a te d detection of a preseizure state based on a decrease in synchronization in intracranial electroencephalogram recordings from epilepsy patients / F. Mormann, R.G. Andrzejak, T. Kreuz, C. Rieke, P. David, C.E. Elger and K. Lehnertz // Physical Review E. — 2003. — 67, N 21912.

28. L o ng-term prospective on-line real-time seizure prediction / L.D. Iasemidis, D.S. Shiau, P.M. Pardalos, W. Chaovalitwongse, K. Narayanan, A. Prasad, K. Tsakalis and P.R. Carney // Clin. Neurophysiol. — 2005. — 116. - P. 532-544.

29. Iasemidis L.D., Principe J.C., Czaplewski J.M., Gilmore R.L., R oper S.N., S a c kellares J.C. Spatiotemporal transition to epileptic seizures: a nonlinear dynamical analysis of scalp and intracranial EEG recordings // In Spatiotemporal Models in Biological and Artificial Systems / Ed. by F.L. Silva, J.C. Principe and L.B. Almeida. Amsterdam: IOS Press. - 1997. — P. 81-88.

30. D y n a m i a 1 resetting of the human brain at epileptic seizures: application of nonlinear dynamics and global optimization techniques / L.D. Iasemidis, D.S. Shiau, J.C. Sackellares, P.M. Pardalos and A. Prasad // IEEE Trans. Biomed. Eng. — 2004. — 51. — P. 493-506.

31. Predictability analysis for an automated seizure prediction algorithm / J.C. Sackellares, D.S. Shiau, J.C. Principe, M.C.K. Yang, L.K. Dance, W. Suharitdamrong, W. Chaovalitwongse, P.M. Pardalos and L.D. Iasemidis // J. of Clinical Neurophysiology. — 2006. — 23. — P. 509-520.

32. Peters T.E., Bhavaraju N.C., Frei M.G., Osorio I. Network system for automated seizure detection and contingent delivery of therapy // J. of Clinical Neurophysiology. 2001. - 18. - P. 545-549.

33. Shiau D. Signal identification and forecasting in nonstationary time series data // $\mathrm{Ph} . \mathrm{D}$. dissertation, University of Florida, 2001.

34. A s s e s s m e n t of a scalp EEG-based automated seizure detection system / K.M. Kelly, D.S. Shiau, R.T. Kern, J.H. Chien, M.C.K. Yang, K.A. Yandora, J.P. Valeriano, J.J. Halford and J.C. Sackellares // Clin. Neurophysiol. — 2010. — 121, N 11. - P. 1832-1843.

35. A feedback control systems view of epileptic seizures / K. Tsakalis, N. Chakravarthy, S. Sabesan, L.D. Iasemidis and P.M. Pardalos // Cybern. Sys. Analysis. — 2006. — 42. P. 483-495.

36. The seizure prediction characteristic: a general framework to assess and compare seizure prediction methods / M. Winterhalder, T. Maiwald, H.U. Voss, R. Aschenbrenner-Scheibe, J. Timmer and A. Schulze-Bonhage // Epilepsy and Behavior. - 2003. — 4 _ - P. 318-325.

37. Testing statistical significance of multivariate time series analysis techniques for epileptic seizure prediction / B. Schelter, M. Winterhalder, T. Maiwald, A. Brandt, A. Schad, A. Schulze-Bonhage and J. Timmer // Chaos: An Interdisciplinary J. of Nonlinear Science. — 2006. — 16, N 013108.

38. Me a s u r e profile surrogates: A method to validate the performance of epileptic seizure prediction algorithms / T. Kreuz, R.G. Andrzejak, F. Mormann, A. Kraskov, H. Stögbauer, C.E. Elger, K. Lehnertz and P. Grassberger // Physical Review E. - 2004. - 69, N 61915.

39. Testing the null hypothesis of the nonexistence of a preseizure state / R.G. Andrzejak, F. Mormann, T. Kreuz, C. Rieke, A. Kraskov, C.E. Elger and K. Lehnertz // Physical Review E. 2003. - 67, N 10901.

40. Zhang J., Xanthopoulos P., Liu C-C., Bearden S., Uthman B.M., Pardalos P.M. Real-time differentiation of nonconvulsive status epilepticus from other encephalopathies using quantitative EEG analysis: a pilot study // Epilepsia. — 2010. — 51(2). P. 243-250.

41. Zhang J., Xanthopoulos P., Chien J-H., Tomaino V., Pardalos P.M. Minimum prediction error models and causal relations between time series // Wiley Encyclopedia of Operations Research and Management Science. — 2011. — 5. - P. 3271-3285.

Поступила 21.01.2011 